\title{
Extended light scattering model incorporating coherence for thin-film silicon solar cells
}

\author{
Thomas Lanz, ${ }^{1, a)}$ Beat Ruhstaller, ${ }^{1,2}$ Corsin Battaglia, ${ }^{3}$ and Christophe Ballif ${ }^{3}$ \\ ${ }^{1}$ Zurich University of Applied Sciences, Institute of Computational Physics, Wildbachstrasse 21 , \\ Winterthur CH-8401, Switzerland \\ ${ }^{2}$ Fluxim AG, Dorfstrasse 7, Feusisberg CH-8835, Switzerland \\ ${ }^{3}$ Ecole Polytechnique Fédérale de Lausanne (EPFL), Institute of Microengineering (IMT), Photovoltaics and \\ Thin Film Laboratory, Rue A.-L. Breguet 2, Neuchătel CH-2000, Switzerland
}

(Received 11 April 2011; accepted 6 July 2011; published online 12 August 2011)

\begin{abstract}
We present a comprehensive scalar light-scattering model for the optical simulation of silicon thin film solar cells. The model integrates coherent light propagation in thin layers with a direct, noniterative treatment of light scattered at rough layer interfaces. The direct solution approach ensures computational efficiency, which is a key advantage for extensive calculations in the context of evaluation of different cell designs and parameter extraction. We validate the model with experimental external quantum efficiency spectra of state-of-the-art microcrystalline silicon solar cells. The simulations agree very well with measurements for cells deposited on both rough and flat substrates. The model is then applied to study the influence of the absorber layer thickness on the maximum achievable photocurrent for the two cell types. This efficient numerical framework will enable a quantitative model-based assessment of the optimization potential for light trapping in textured thin film silicon solar cells. (C) 2011 American Institute of Physics. [doi:10.1063/1.3622328]
\end{abstract}

\section{INTRODUCTION}

To reach high conversion efficiencies in thin film silicon solar cells, advanced light trapping mechanisms need to be employed. ${ }^{1}$ To increase the optical absorption in the cells and thus enhance the short circuit current density, light scattering is induced by introducing rough substrate textures. ${ }^{2-4}$ The combination of multiple layers having thicknesses on different length scales with scattering layer interfaces makes thin film silicon solar cells complex optical devices. Numerical simulations lend themselves to device optimization and can provide quantities that are not accessible in the experiment. Full-wave approaches that rigorously solve Maxwell's equations ${ }^{5,6}$ are very demanding in terms of computing time and memory and are therefore often restricted to small simulation domains or periodic structures. ${ }^{7}$ Alternative approaches take into account the averaged light scattering properties of the rough interfaces as predicted, e.g., by the scalar scattering theory. ${ }^{8-10}$

In this contribution, we present a new approach for the optical modeling of thin film solar cells that is based on the net-radiation method developed by Santbergen. ${ }^{11-13}$ We have extended the net-radiation method to include coherent treatment of thin layers or arbitrary stacks of thin layers. Our paper is organized as follows: in section II, we present the details of the optical model. First, we describe the treatment of incoherent layers and rough interfaces. Then, we discuss the extension to include coherent layers and how to evaluate the layer absorbances. In section III, we apply the model to microcrystalline silicon solar cells by first presenting four simulation cases that illustrate the capabilities of the model.

\footnotetext{
a) Author to whom correspondence should be addressed. Electronic mail: thomas.lanz@zhaw.ch.
}

Then, we validate the model with experimental data from state-of-the-art microcrystalline silicon solar cells. Section IV concludes.

\section{METHODS: OPTICAL MODEL}

In this section, we introduce the foundations of the optical model. The physical problem that we aim to solve is to determine, for a given solar cell structure and incident illumination, the spectral absorption profile throughout the cell. The input parameters are the thicknesses of all layers, the refractive index dispersions of all materials, and the scattering properties of all interfaces (as explained below), as well as the illumination spectrum. The quantities that we obtain from the calculation are the reflection and transmission and the layer absorbances of all layers as a function of the wavelength. The thin film solar cell is represented by a stack of homogeneous layers. We distinguish between incoherent and coherent layers. In incoherent layers, only the intensity of the light is considered. This is appropriate for layers that are thicker than the coherence length of the incident illumination and thus do not allow for coherent propagation of light. In thin, coherent layers, a transfer-matrix formalism is applied to take account of the wave nature of the light. The dispersion of the refractive indices of the materials is considered, and the calculations are performed separately for each wavelength. In contrast to ray-tracing approaches, ${ }^{14}$ our model solves directly for the complete steady-state intensity distribution instead of iteratively building it up. Thus, our approach is computationally efficient. Our approach is also in contrast to the work of Krč et al., ${ }^{9}$ where only the scattered light is treated iteratively, whereas in our model, both direct and scattered light distributions are computed noniteratively. Furthermore, in thin layers, we consider coherent 
propagation of both direct and scattered light. The boundary conditions between coherent and incoherent layers are formulated using conservation of energy. The model is implemented in Wolfram Mathematica and runs on a standard personal computer. In the following, we present the details of the optical model.

\section{A. Incoherent layers and rough interfaces}

In incoherent layers, only the intensity of the light is taken into account. To this end, at every interface, we define four flux vectors denoted by subscripts $a, b, c$, and $d$, as depicted in Fig. 1. The intensity distribution between $0^{\circ}$ (normal to the interface) and $90^{\circ}$ is described by a vector such as $\boldsymbol{q}_{i, a}=\left(q_{i, a}^{1}, q_{i, a}^{2}, q_{i, a}^{3}, \ldots, q_{i, a}^{D}\right)$, where $D$ is the number of discrete angles. Propagation through the layers and reflection and transmission at the interfaces connect the flux vectors. The following equations specify the relations between the flux vectors: ${ }^{11}$

$$
\begin{aligned}
& \boldsymbol{q}_{i, a}=\tau_{i-1} \times \boldsymbol{q}_{i-1, d} \\
& \boldsymbol{q}_{i, b}=r_{i,+} \times \boldsymbol{q}_{i, a}+t_{i,-} \times \boldsymbol{q}_{i, c} \\
& \boldsymbol{q}_{i, c}=\tau_{i} \times \boldsymbol{q}_{i+1, b} \\
& \boldsymbol{q}_{i, d}=r_{i,-} \times \boldsymbol{q}_{i, c}+t_{i,+} \times \boldsymbol{q}_{i, a} .
\end{aligned}
$$

$\tau_{i}, r_{i}$, and $t_{i}$ are square matrices with dimension $D \times D$. The matrix $\tau_{i}$ is diagonal and describes the intensity propagation inside layer $i$ from interface $i$ to interface $i+1$ or in the opposite direction. Its entries are given by

$$
\tau_{i}^{(m, m)}=\exp \left(-\alpha d_{i} / \cos \theta_{m}\right),
$$

where $\alpha=4 \pi k / \lambda$.

Here, $\alpha$ denotes the absorption coefficient that can be determined from the extinction coefficient $k$, the imaginary

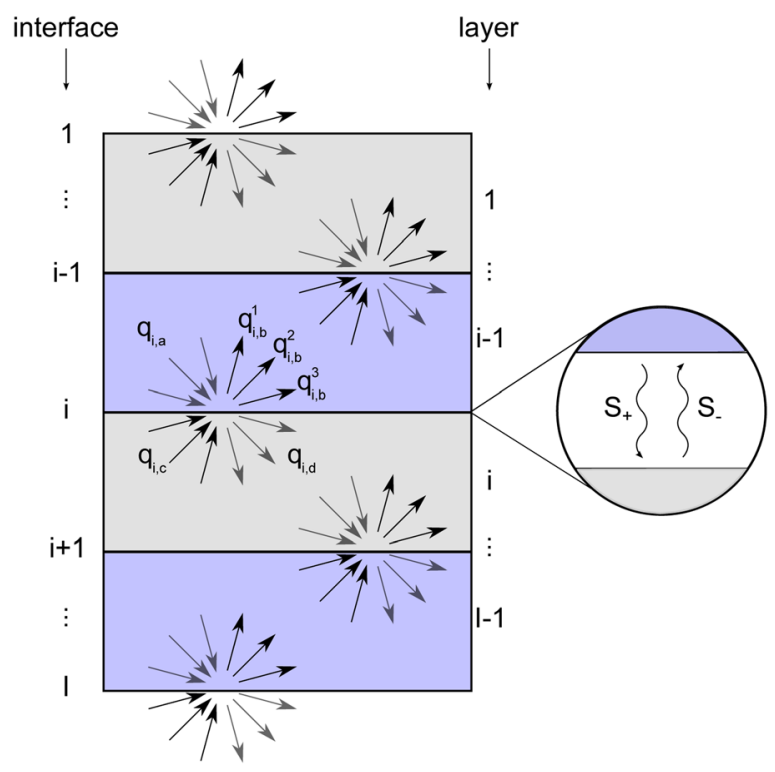

FIG. 1. (Color online) The flux vectors that describe the angular distribution of the total light intensity at every interface. The inset on the right illustrates the wave propagation in thin layers in upward $\left(S_{-}\right)$and downward $\left(S_{+}\right)$ direction. Figure adapted from Santbergen [Ref. 15]. part of the refractive index $N=n+i k$. The thickness of layer $i$ is denoted by $d_{i}$, and thus $d_{i} / \cos \theta_{m}$ is the distance that light with a propagation angle of $\theta_{m}$ (measured from the normal to the interfaces) traverses between the two interfaces. The matrices $r_{i}$ and $t_{i}$ describe the reflection at and the transmission through the interface, where the plus (e.g., $r_{i,+}$ ) denotes incidence from above the interface and the minus sign from below. For flat interfaces, only specular reflection occurs, i.e., angle of reflection equals angle of incidence and $r_{i}$ is a diagonal matrix. For rough interfaces that scatter light, $r_{i}$ and $t_{i}$ describe the angular distribution of the scattered light. For light, e.g., that is incident with an angle $\theta_{m}$, the distribution of the light that is scattered in reflection is described by the mth column of the matrix $r_{i}$.

To determine the amount of scattered light, we employ the scalar scattering theory. ${ }^{16}$ It provides an analytical formula for the spectral dependence of the haze in transmission, which is defined as the ratio between the diffusely scattered and the total light intensity, given by

$H_{T}(\lambda)=T_{\text {diff }} / T_{\text {tot }} \approx 1-\exp \left[-\left(\frac{2 \pi \sigma_{\mathrm{rms}}\left|n_{1}-n_{2}\right| c_{T}(\lambda)}{\lambda}\right)^{2}\right]$.

In Eq. (3), we consider normal incidence and have included a correction function $c_{T}(\lambda)$, which has been proposed for the projection of light scattering at internal interfaces. ${ }^{9} n_{1}$ and $n_{2}$ denote the refractive indices of the incident and the adjacent medium, $\sigma_{\mathrm{rms}}$, denotes the root-mean-square (rms) roughness of the interface. Dominé et al. ${ }^{17}$ propose to calculate the angular distribution and the fraction of the scattered light based on measured surface profiles of the interfaces. Their procedure is compatible with our approach, and we envisage to employ their formalism as an input to our model; this, however, is beyond the scope of this study. In the present study, we employ the analytical model proposed by Phong ${ }^{18}$ for the angular distribution function. It is well-suited to characterize our model without introducing unnecessary complexity. The distribution $I(\theta)$ of the scattered light is given by

$$
I(\theta)=c \times \cos ^{l}\left(\theta-\theta_{\text {spec }}\right),
$$

where $l$ is the Phong exponent, c is a normalization constant, and $\theta_{\text {spec }}$ is the direction of specular reflection or transmission. The case $l=1$ corresponds to Lambertian scattering, whereas the distribution becomes more and more specular for higher values of $l$. The normalization constant is used to normalize the total scattered intensity, where we make the assumption that the same fraction of light is reflected from a rough interface as from a flat interface. Total transmission and total reflection add up to 1 .

Physically, light scattering is not confined to the plane of incidence, but results in scattering cones. To take this into account, in one-dimensional modeling it has been suggested $^{19}$ to transform the angular distribution functions as $I_{\text {corr }}(\theta)=c \times I(\theta) \times \sin \theta$. The factor $\sin \theta$ results from the projection of the three-dimensional scattering cone onto the plane of incidence, where we have assumed rotational 
symmetry. The distribution functions in our model accommodate both the haze according to Eq. (3) and the distribution of scattered light by distinguishing between a specular part and a diffusely scattered part. For normal incidence, we denote the angular range from $0^{\circ}$ to $3^{\circ}$ as the specular part.

\section{B. Coherent layers}

In thin layers, the wave nature of light becomes apparent and interference effects may arise. To take this into account, we employ a transfer-matrix method. ${ }^{20}$ Individual thin layers or stacks of thin layers are represented by a flat multilayer structure. The transfer-matrix method allows calculating the reflection from and the transmission through this multilayer structure for all angles of incidence. One can thus define reflection and transmission matrices for this sub-stack as for an interface between two incoherent layers, cf. Eq. (1). By describing a stack of coherent layers as an interface, the complete structure is thus reduced to a sequence of incoherent layers. Figure 1 illustrates this concept. $S_{-}$and $S_{+}$denote the Poynting vectors of the upward and downward propagating waves in the coherent sub-stack.

\section{Evaluation of layer absorbance spectra}

Once the equations for all the interfaces have been established, as described in Subsections II A and II B, they are adjusted to the boundary conditions. This concerns the fluxes $q_{1, a}$ and $q_{N, c}$, i.e., the fluxes incident on the front and the back of the structure. If the solar cell is illuminated only from the front side under an incidence angle $\theta_{i n c}$, then the corresponding element in $q_{1, a}$ is set to one and all other entries to zero and all entries in $q_{N, c}$ are set to zero. The set of equations is assembled to one matrix equation, which can be solved for the unknown fluxes. The total (specular plus diffuse) reflection is then given by $\mathrm{R}=\sum_{m=1}^{D} q_{1, b}^{m}$ and the transmission, correspondingly, by $\mathrm{T}=\sum_{m=1}^{D} q_{N, d}^{m}$. One may also distinguish between specular and diffuse reflection, which may turn useful for the validation with experiments. The absorption in the incoherent layer between interfaces $i$ and $i+1$ is given by

$$
A_{i, \text { incoh }}(\lambda)=\sum_{m=1}^{D} q_{i, d}^{m}-q_{i+1, a}^{m}+q_{i+1, b}^{m}-q_{i, c}^{m} .
$$

To calculate the absorption in coherent layers, we can use the Poynting vector (energy flux) that is calculated in the transfer-matrix method. While the reflectivity and transmissivity of the coherent stack is independent of the incident intensity, the Poynting vector needs to be normalized according to the intensity that is incident on the stack. Once the solution for the intensity fluxes is known, the magnitude of the Poynting vector can be determined using conservation of energy. For the case of a single coherent layer and perpendicular propagation, these relations can be expressed as

$$
\begin{aligned}
& q_{i, a}^{1}-q_{i, b}^{1}-\gamma \times \tilde{S}_{+}(\text {top })+\delta \times \tilde{S}_{-}(\text {top })=0 \\
& q_{i, c}^{1}-q_{i, d}^{1}+\gamma \times \tilde{S}_{+}(\text {bottom })-\delta \times \tilde{S}_{-}(\text {bottom })=0 .
\end{aligned}
$$

Here, $\tilde{S}_{+}(x)$ denotes the unscaled energy flux in forward direction at location $x$ inside the coherent layer and $\tilde{S}_{-}(x)$

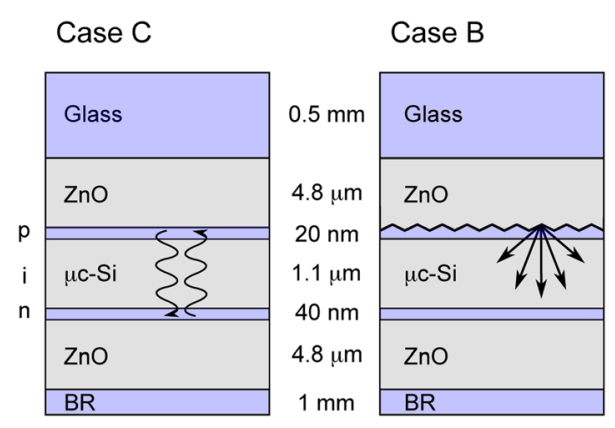

FIG. 2. (Color online) Illustration of two considered simulation cases $\mathrm{C}$ (left) and B (right). See Table I for further details.

correspondingly denotes the energy flux in backward direction. $\gamma$ and $\delta$ are the unknown constants used to scale the energy fluxes. Equation (6) can be solved for the two constants $\gamma$ and $\delta$ to obtain $S_{+}(x)=\gamma \times \tilde{S}_{+}(x)$ and $S_{-}(x)=\delta \times \tilde{S}_{-}(x)$. Both the Poynting vectors in forward and backward direction are continuous throughout the stack of coherent layers. The absorption in one coherent layer then amounts to

$$
A_{\mathrm{coh}}(\lambda)=S_{+}(\text {top })-S_{+}(\text {bottom })+S_{-}(\text {bottom })-S_{-}(\text {top }) \text {. }
$$

The maximum achievable photocurrent is obtained from the layer absorbance of the intrinsic layer

$$
I_{\text {photo }}=\frac{e}{h c} \int A_{\text {int }}(\lambda) \times \Phi(\lambda) \times \lambda d \lambda,
$$

where $e$ denotes the elementary charge, $h$ Planck's constant, $c$ the speed of light, and $\Phi(\lambda)$ the illumination spectrum.

\section{RESULTS AND DISCUSSION}

\section{A. Experimental}

We use two types of microcrystalline silicon solar cells in the $p-i-n$ configuration for the analysis with our optical model. The preparation and experimental characterization of the cells have been described in detail in Refs. 21-23. As front and back electrode, both cells use lightly doped $\mathrm{ZnO}$ deposited by low-pressure chemical vapor deposition with a thickness of $4.8 \mu \mathrm{m}$. During growth, $\mathrm{ZnO}$ develops a sharp pyramidal surface structure; to make it suitable for the deposition of $\mu \mathrm{c}-\mathrm{Si}$, it is necessary to subject it to a plasma treatment. ${ }^{24}$ For the first cell, subsequently denoted as the rough cell, a 20 min plasma treatment is applied to the $\mathrm{ZnO}$, resulting in a mean surface roughness of $150 \mathrm{~nm} .{ }^{17}$ For the second cell, subsequently denoted as the flat cell, the front electrode is completely flattened by chemical-mechanical polishing.

\begin{tabular}{|c|c|c|c|c|}
\hline & Case A & Case B & Case C & Case D \\
\hline$\mu \mathrm{c}-\mathrm{Si}$ layer & Coherent & Incoherent & Coherent & Incoherent \\
\hline $\mathrm{ZnO} / \mu \mathrm{c}-\mathrm{Si}$ interface & Scattering & Scattering & Flat & Flat \\
\hline
\end{tabular}
The p-i-n microcrystalline stack is prepared by plasmaenhanced chemical vapor deposition (PECVD) with thicknesses of $20 \mathrm{~nm}$ (p), $1100 \mathrm{~nm}$ (i), and $40 \mathrm{~nm}$ (n). The p layer

TABLE I. Simulation cases considered in the calculations. 


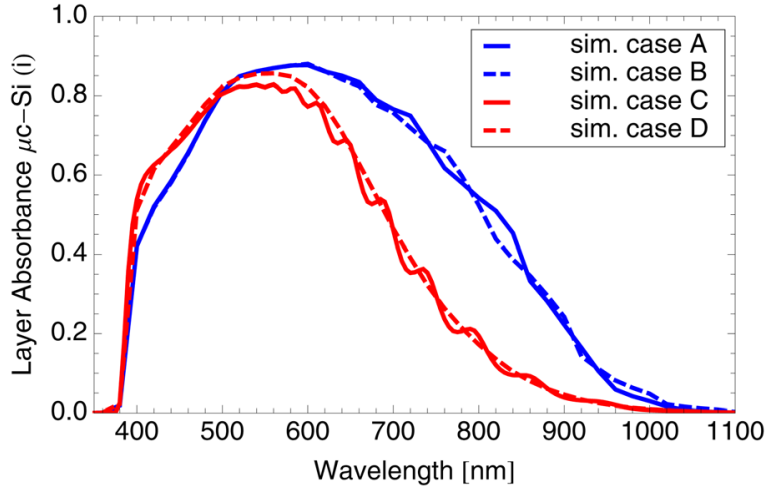

FIG. 3. (Color online) Calculated absorbance in the intrinsic microcrystalline silicon layer of the four simulation cases.

consists of amorphous silicon oxide containing nanocrystalline silicon grains $\left(\mathrm{nc}-\mathrm{SiO}_{\mathrm{x}}\right)$. A white dielectric reflector is applied on the back contact on both cells. Refractive indices have been determined by spectroscopic ellipsometry measurements. The AM1.5 short circuit currents are $J_{s c \text { flat }}=16.22 \mathrm{~mA} / \mathrm{cm}^{2}$ and $J_{s c, \text { rough }}=23.15 \mathrm{~mA} / \mathrm{cm}^{2}$.

\section{B. Simulation results}

For the optical simulation of the $\mu \mathrm{c}-\mathrm{Si}$ solar cells described above, we define four simulation cases with distinct sets of input parameters for the optical model. The layer thicknesses are the same for all cases and are given in Fig. 2. We differentiate between a coherent and incoherent absorber layer and between a scattering or flat $\mathrm{ZnO} / \mu \mathrm{c}$-Si front interface and the other interfaces are considered to be flat in all cases; Table I specifies the four cases and Fig. 2 illustrates two of them. For the scattering interface, Eq. (3) is used to determine the Haze in transmission, and a Lambertian distribution of the scattered light is assumed $(l=1$ in Eq. (4)). For the correction factor $c_{T}(\lambda)$, which is the only free parameter in the model, we find a constant value of 4.7 to result in a good agreement with the measurements (for the validation with measurement data, see Subsection III C). This correction is most likely necessary, due to the simplifying assumption that only one interface in the structure is scattering. The

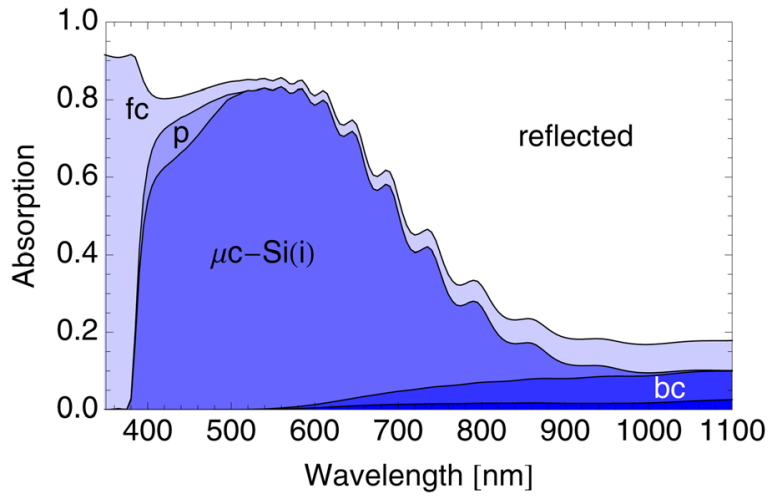

FIG. 4. (Color online) Area plot of the fractional absorbance for each layer of the $\mu \mathrm{c}-\mathrm{Si}$ solar cell, simulation case C. fc: $\mathrm{ZnO}$ front contact; p: p-nc$\mathrm{SiO}_{x}$; bc: $\mathrm{ZnO}$ back contact.

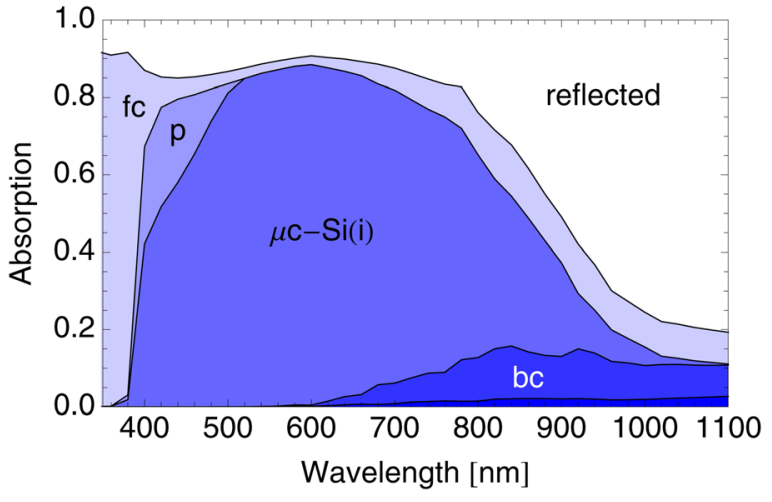

FIG. 5. (Color online) Area plot of the fractional absorbance for each layer of the $\mu \mathrm{c}-\mathrm{Si}$ solar cell, simulation case B. fc: $\mathrm{ZnO}$ front contact, p: p-nc$\mathrm{SiO}_{x}$; bc: $\mathrm{ZnO}$ back contact.

white dielectric reflector has been reported to produce similar results as a silver layer. ${ }^{25}$ To simplify the analysis, we therefore approximate the refractive index of the dielectric by that of silver.

Figure 3 compares the calculated absorbance in the intrinsic microcrystalline silicon layer for the four simulation cases. For short wavelengths below 500 nm, scattering leads to increased (parasitic) absorption in the p-doped layer, reducing the absorption in the intrinsic layer (cases $\mathrm{A}$ and B), as is shown below in Figs. 4 and 5. For wavelengths above $500 \mathrm{~nm}$, scattering increases the absorption, due to enhanced light trapping. Cases $\mathrm{A}$ and $\mathrm{B}$ yield almost the same absorption as the distribution of propagation directions produced by scattering averages out of the effect of coherence. This is a confirmation that we have a consistent implementation of the boundary conditions between coherent and incoherent layers. For the flat case $\mathrm{C}$, the coherent treatment of the absorber layer results in strong interference effects in the absorption spectrum. These four simulation cases illustrate the effect of coherence and the quantitative enhancement of the absorption due to a single scattering interface.

Figures 4 and 5 present a detailed analysis of the layer absorbances for the simulation cases $\mathrm{C}$ and $\mathrm{B}$. As the cells are opaque, there is no transmission through the cells, and the layer absorbances together with the reflection add up to one. For both cases, we find only negligible absorbance in the thin n-doped

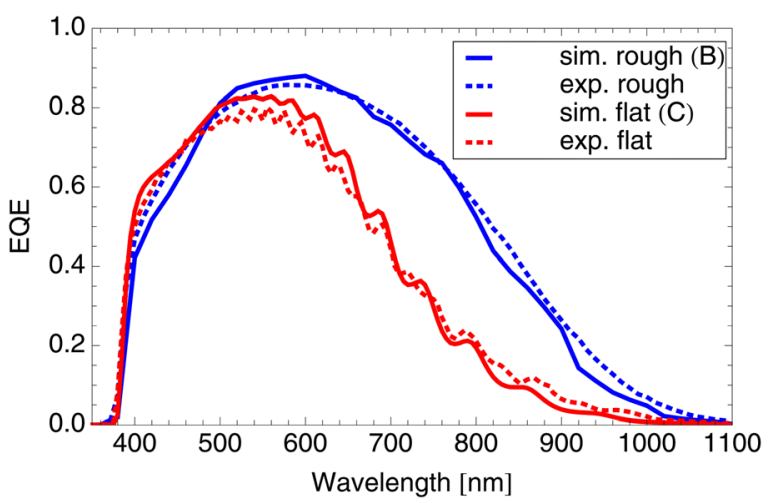

FIG. 6. (Color online) Comparison of simulated (sim.) and experimental (exp.) external quantum efficiency (EQE) spectra. 


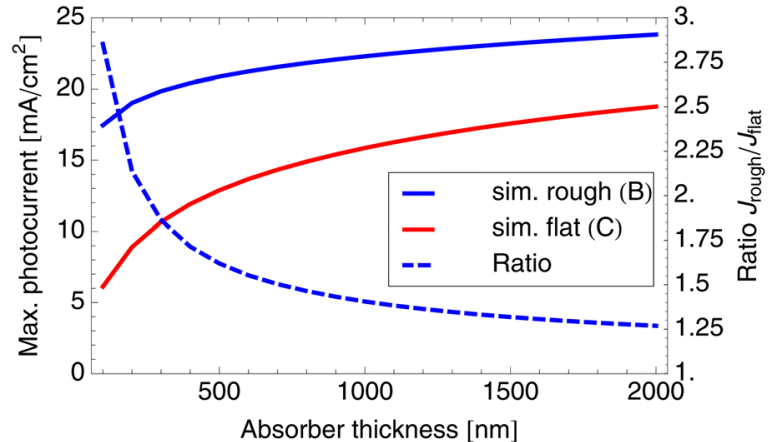

FIG. 7. (Color online) Influence of the absorber thickness on the maximum achievable photocurrent. The dashed curve illustrates the relative gain that can be achieved by scattering.

layer. Figure 5 illustrates the increased parasitic absorbance induced by scattering in the front and back contact and the p-doped layer and the overall strong reduction in reflection.

\section{Validation of the model}

After demonstrating the feasibility of the extended optical model for simulating thin film silicon solar cells, we now turn to the model validation with experimental data. Figure 6 compares the calculated absorbance in the intrinsic layer for the cases $\mathrm{B}$ and $\mathrm{C}$ with the measured quantum efficiency of the two solar cells. We thus assume ideal photon-to-charge conversion and extraction of charge carriers. Overall, there is a very good agreement between simulation and measurement. Simulation case $\mathrm{C}$ follows closely the measured, oscillating EQE spectrum of the flat cell. Simulation case B follows the measured EQE spectrum of the cell with the rough interface. Between 400 and $500 \mathrm{~nm}$, the simulated EQE is smaller than the experimental one, likely due to an overestimation of the absorbance in the pdoped layer in our model (cf. Fig. 5). As we consider a single rough interface in this simulation case, the calculated increased absorption may be attributed to the scattering at the $\mathrm{ZnO} / \mu \mathrm{c}-\mathrm{Si}$ interface. A single scattering interface with enhanced scattering properties, as detailed above, may thus produce the same absorption enhancement as multiple rough interfaces. The maximum photocurrents determined from the simulated absorbance spectra are $J_{\text {photo flat }(C)}=16.63 \mathrm{~mA} / \mathrm{cm}^{2}$ (measured: $J_{s c, f l a t}=16.22 \mathrm{~mA} / \mathrm{cm}^{2}$ ) and $J_{\text {photo, rough }(B)}=22.68 \mathrm{~mA} / \mathrm{cm}^{2}$ (measured: $J_{s c, \text { rough }}=23.15 \mathrm{~mA} / \mathrm{cm}^{2}$ ).

Figure 7 presents an analysis of the influence of the thickness of the intrinsic microcrystalline silicon absorber layer on the maximum achievable photocurrent for the simulation cases B (with scattering) and C (flat, no scattering). For all thicknesses, scattering leads to an increased photocurrent. However, the gain in current due to scattering is higher for smaller absorber thicknesses, which is illustrated by the ratio $J_{\text {rough }} / J_{\text {flat }}$ of the two currents, plotted in the same figure. At an absorber layer thickness of $2000 \mathrm{~nm}$, the current gain due to scattering is down to $25 \%$. It has been reported ${ }^{22}$ that, for cell thicknesses above $4000 \mathrm{~nm}$, the photocurrent saturates for this type of cells deposited on the rough substrate due to electrical charge collection problems. It therefore seems reasonable to assume that, in the range studied here, these effects can be neglected.

\section{CONCLUSIONS}

We presented a numerical model for the optical fulldevice simulation of thin film solar cells. The model incorporates coherent and incoherent light propagation as well as scattering at rough layer interfaces. On a standard personal computer, the simulation of an entire spectrum takes about 10 seconds. The solution procedure of the model, as well as the underlying equations, were presented in detail. The interplay of scattering and coherence was demonstrated using four simulation cases. By calculating the absorbance in every layer of the solar cell structure, the model allows for an assessment of the parasitic absorbance in non-active layers. We validated the model using measurements of external quantum efficiency spectra of microcrystalline silicon solar cells. For cells on both rough and flat substrates, measurements and simulations agree very well. This agreement and the fact that interference fringes are present in the flat case illustrates the necessity of a model that unites coherence, incoherence, and scattering for simulating various cell types. The model was then applied to study the influence of the absorber thickness on the maximum achievable photocurrent. We confirmed a strong enhancement, due to scattering for thin absorber layers. The computational efficiency of our model will allow for a systematic simulation-based device optimization, such as the choice of substrate topology, which influences both current enhancement and device stability.

\section{ACKNOWLEDGMENTS}

We thankfully acknowledge P. Cuony for performing spectroscopic measurements. The work was supported by Swiss Electric Research in the thinPV and DURSOL CCEM projects.

${ }^{1}$ A. V. Shah, M. Vanecek, J. Meier, F. Meillaud, J. Guillet, D. Fischer, C. Droz, X. Niquille, S. Faÿ, E. Vallat-Sauvain, V. Terrazzoni-Daudrix, and J. Bailat, J. Non-Cryst. Solids 338-340, 639 (2004).

${ }^{2}$ H. Iida, N. Shiba, T. Mishuku, H. Karasawa, A. Ito, M. Yamanaka, and Y. Hayashi, IEEE Electron Device Lett. 4, 157 (1983).

${ }^{3}$ H. Sai and M. Kondo, J. Appl. Phys. 105, 094511 (2009).

${ }^{4}$ T. Söderström, F. J. Haug, X. Niquille, and C. Ballif, Prog. Photovoltaics 17, 165 (2009).

${ }^{5}$ C. Rockstuhl, F. Lederer, K. Bittkau, and R. Carius, Appl. Phys. Lett. 91, 171104 (2007).

${ }^{6}$ P. Obermeyer, C. Haase, and H. Stiebig, Appl. Phys. Lett. 92 (2008).

${ }^{7}$ A. Naqavi, K. Söderström, F.-J. Haug, V. Paeder, T. Scharf, H. P. Herzig, and C. Ballif, Opt. Express 19, 128 (2011).

${ }^{8}$ A. Poruba, A. Fejfar, Z. Remes, J. Springer, M. Vanecek, J. Kocka, J. Meier, P. Torres, and A. Shah, J. Appl. Phys. 88, 148 (2000).

${ }^{9}$ J. Krč, M. Zeman, F. Smole, and M. Topič, J. Appl. Phys. 92, 749 (2002).

${ }^{10}$ J. Krč, F. Smole, and M. Topič, Prog. Photovoltaics 11, 429 (2003).

${ }^{11}$ R. Santbergen and R. J. C. van Zolingen, Energy Convers. Manage. 47, $3572(2006)$

${ }^{12} \mathrm{~T}$. Lanz and B. Ruhstaller, in Advanced Photonics/Renewable Energy: OSA Optics \& Photonics Congress, Karlsruhe, Germany, 21-24 June 2010.

${ }^{13}$ T. Lanz, B. Ruhstaller, C. Battaglia, F.-J. Haug, and C. Ballif, in 25th European Photovoltaic Solar Energy Conference and Exhibition, Valencia, Spain, 6-10 September 2010.

${ }^{14}$ D. Thorp and S. Wenham, Sol. Energy Mater. Sol. Cells 48, 295 (1997).

${ }^{15}$ R. Santbergen, J. Goud, M. Zeman, J. van Roosmalen, and R. van Zolingen, Sol. Energy Mater. Sol. Cells 94, 715 (2010).

${ }^{16}$ C. Carniglia, Opt. Eng. 18, 104 (1979). 
${ }^{17}$ D. Domine, F. J. Haug, C. Battaglia, and C. Ballif, J. Appl. Phys. 107, 044504 (2010).

${ }^{18}$ B. T. Phong, Commun. ACM 18, 311 (1975).

${ }^{19} \mathrm{~J}$. Krč, "Analysis and modelling of thin-film optoelectronic structures based on amorphous silicon," Ph.D. thesis, (University of Ljubljana, 2003).

${ }^{20} \mathrm{P}$. Yeh, Optical Waves in Layered Media (Wiley, New York, 2005).

${ }^{21}$ G. Bugnon, A. Feltrin, F. Meillaud, J. Bailat, and C. Ballif, J. Appl. Phys. 105, 064507 (2009)

${ }^{22}$ M. Boccard, P. Cuony, M. Despeisse, D. Dominé, A. Feltrin, N. Wyrsch, and C. Ballif, Sol. Energy Mater. Sol. Cells 95, 195 (2011).
${ }^{23}$ P. Cuony, M. Marending, D. T. L. Alexander, M. Boccard, G. Bugnon, M. Despeisse, and C. Ballif, Appl. Phys. Lett. 97, 213502 (2010).

${ }^{24}$ J. Bailat, L. Fesquet, J.-B. Orhan, B. Wolf, P. Madliger, J. Steinhauser, S. Benagli, D. Borrello, L. Castens, G. Monteduro, M. Marmelo, B. Dehbozorghi, E. Vallat-Sauvain, X. Multone, D. Romang, J.-F. Boucher, J. Meier, U. Kroll, M. Despeisse, G. Bugnon, C. Ballif, S. Marjanovic, G. Kohnke, N. Borrelli, K. Koch, J. Liu, R. Modavis, D. Thelen, S. Vallon, A. Zakharian, and D. Weidman, in Proceedings of the 25th EU-PVSEC and 05th WC-PEC Conference, Valencia, Spain, 6-10 September 2010.

${ }^{25}$ B. Lipovšek, J. Krč, O. Isabella, M. Zeman, and M. Topič, J. Appl. Phys. 108, 103115 (2010) 